\title{
Editorial: Multiparametric Evaluation Predicts Different Mid-Term Outcomes in Crohn's Disease
}

\author{
Tim Raine \\ Department of Gastroenterology, Addenbrooke's Hospital, Cambridge University Teaching Hospitals NHS \\ Foundation Trust, Cambridge, UK
}

Traditional models of the medical management of Crohn's disease (CD) can be characterised as "sequential failure", involving step-wise escalation through a well-established sequence of therapies driven by the breakthrough appearance of clinical symptoms of disease. While the clinical assessment of patients remains the bedrock of $\mathrm{CD}$ care, we are increasingly shifting paradigms towards the assessment of multiple indicators of disease activity, including objective markers of inflammation, as we strive to improve outcomes [1]. Appropriate and timely identification of the patient in need of treatment escalation or change may help to mitigate the patient distress associated with the use of ineffective treatments, as well as the risk and cost associated with such treatments. However, the physician is currently faced with a range of potential assessment modalities, the relative importance of which remains unclear.

In this edition of Digestive Diseases, Laterza et al. [2] have set out to evaluate the implications of simultaneous assessments on patients with CD using 3 indices: clinical scoring, endoscopy and CT enterography (CTE). The authors identify a cohort of 57 patients undergoing baseline assessment using all 3 methods within 1 month, with clin-

\section{KARGER}

(c) 2018 S. Karger AG, Basel

E-Mail karger@karger.com

www.karger.com/ddi ical follow-up to 36 months in all except 4 cases. Tellingly, correlation between the assessment methods was poor, with most patients displaying discordance between clinical assessment and assessment by CTE or endoscopy. Correlation between endoscopy and CTE was scarcely better, with agreement still in less than half of cases and with the majority of patients in endoscopic remission showing evidence of ongoing transmural inflammation on CTE. Importantly, patients where all 3 modalities indicated remission were significantly less likely to undergo hospitalisation during follow-up, while patients with endoscopic activity were more likely to require treatment escalation and patients with transmural inflammation were more likely to require hospitalisation. For those patients where clinical and endoscopic assessments were discrepant, inflammatory activity as assessed on CTE correlated with a numeric trend towards the need for treatment escalation or hospitalisation that did not reach statistical significance.

In this retrospective study, by selecting a cohort who were determined by their treating clinicians to need these assessments in a narrow time window, Laterza et al. [2] have of course, identified a difficult cohort of patients 
who may well have more complex disease as well as a higher baseline probability of discordance between assessment modalities. It is notable, for example, that most patients exhibited stricturing disease behaviour. But it is in precisely these difficult cohorts of patients where we need to improve our understanding of the use and performance of these investigations.

Most of the outcomes measured occurred relatively soon after the point of initial assessment, suggesting that these tests were informing shorter-term treatment decisions rather than offering longer-term prognostic value. Likewise, it would be interesting to know the extent to which biochemical assessment (C-reactive protein, faecal calprotectin) and histologic activity might have correlated with findings and predicted outcomes in this cohort. Finally, the use of CTE rather than MRI necessitated the use of an unvalidated scoring system and may not reflect contemporary practice but still provides a useful assessment of transmural inflammation.

What lessons can we draw from this interesting study? Certainly no one single modality is currently best placed to predict outcomes, but we need to consider the cumulative burden of evidence for disease activity using a range of tools. Upon detection of signals of inflammation, what action should the clinician take? Treatment decisions based upon targeting markers of inflammation can impact endoscopic outcomes [1], and improved endoscopic outcomes correlate with improved clinical outcomes [3]. The ongoing REACT2 study should help to close the link between endoscopically informed treatment decisions and clinical outcomes (ClinicalTrials.gov Identifier NCT01698307; results not yet available), but in more difficult patients, similar to the ones included in the study by Laterza et al. [2], we may need to extend our assessments beyond the mucosa and consider the cumulative disease burden using a range of assessment modalities.

\section{Disclosure Statement}

The author has no conflict of interest to declare.

\section{References}

1 Colombel JF, Panaccione R, Bossuyt P, Lukas M, Baert F, Vanasek T, Danalioglu A, Novacek G, Armuzzi A, Hebuterne X, Travis S, Danese S, Reinisch W, Sandborn WJ, Rutgeerts P, Hommes D, Schreiber S, Neimark E, Huang B, Zhou Q, Mendez P, Petersson J, Wallace K, Robinson AM, Thakkar RB, D'Haens G: Effect of tight control management on Crohn's disease (CALM): a multicentre, randomised, controlled phase 3 trial. Lancet 2018;390:2779-2789.

2 Laterza L, Poscaglia A, Minordi L, et al: Multiparametric evaluation predicts different long-term outcomes in Crohn's disease. Dig Dis $2018 ; 36$.
Schnitzler F, Fidder H, Ferrante M, Noman M, Arijs I, Van Assche G, Hoffman I, Van Steen K, Vermeire S, Rutgeerts P: Mucosal healing predicts long-term outcome of maintenance therapy with infliximab in Crohn's disease. Inflamm Bowel Dis 2009;15:12951301. 